$$
0<t-x<1 / n \text { implies }[F(t)-F(x)] /(t-x) \leqq n ;
$$

the remainder of the proof is unaltered. The next lemma is a slight generalization of a theorem of Marcinkiewicz.

LEMma 5.2. If $f(x)$ is measurable on $[a, b]$, and has either a left major or a right major, and also has either a left minor or a right minor, then $f(x)$ is Perron integrable on $[a, b]$.

The proof is that given by Saks, op. cit., p. 253; the principal change is that the reference to his Theorem 10.1 is replaced by a reference to our Lemma 5.1.

Since every $P^{*}$-integrable function $f(x)$ is measurable and has right majors and right minors, it is also Perron integrable by Lemma 5.2, and the equivalence of the integrals is established.

UNIVERSITY OF VIRGINIA

\title{
ON THE LEAST PRIMITIVE ROOT OF A PRIME
}

\section{LOO-KENG HUA}

It was proved by Vinogradow ${ }^{1}$ that the least positive primitive root $g(p)$ of a prime $p$ is $O\left(2^{m} p^{1 / 2} \log p\right)$ where $m$ denotes the number of different prime factors of $p-1$. In $1930 \mathrm{he}^{2}$ improved the previous result to

$$
g(p)=O\left(2^{m} p^{1 / 2} \log \log p\right)
$$

or more precisely,

$$
g(p) \leqq 2^{m} \frac{p-1}{\phi(p-1)} p^{1 / 2}
$$

It is the purpose of this note, by introducing the notion of the average of character sums, ${ }^{3}$ to prove that if $h(p)$ denotes the primitive root with the least absolute value, $\bmod p$, then

$$
|h(p)|<2^{m} p^{1 / 2}
$$

Received by the editors December 3, 1941.

${ }^{1}$ See, Landau, Vorlesungen über Zahlentheorie, vol. 2, part 7, chap. 14. The original papers of Vinogradow are not available in China.

${ }^{2}$ Comptes Rendus de l'Académie des Sciences de l'URSS, 1930, pp. 7-11.

${ }^{3}$ The present note may be regarded as an introduction of a method which has numerous applications. 
and that for $p \equiv 1(\bmod 4)$, we have

$$
g(p)<2^{m} p^{1 / 2}
$$

while, for $p \equiv 3(\bmod 4)$, we have

$$
g(p)<2^{m+1} p^{1 / 2}
$$

Since

$$
\frac{p-1}{\phi(p-1)} \geqq 2,
$$

the result is always better than that due to Vinogradow.

Lemma 1. Let $p>2,1 \leqq A<p$. For each non-principal character ${ }^{4}$ $\chi(n), \bmod p$, we have

$$
\frac{1}{A+1}\left|\sum_{a=0}^{A} \sum_{n=-a}^{a} \chi(n)\right| \leqq p^{1 / 2}-\frac{A+1}{p^{1 / 2}} .
$$

Proof. Let $\epsilon=e^{2 \pi i / p}$ and let

$$
\tau(\chi)=\sum_{h=1}^{p-1} \chi(h) \epsilon^{h} .
$$

It is known that

$$
|\tau(\chi)|=p^{1 / 2}
$$

For $p \nmid n$, we have

$$
\begin{aligned}
\sum_{h=1}^{p-1} \bar{\chi}(h) \epsilon^{h n} & =\chi(n) \sum_{h=1}^{p-1} \bar{\chi}(h n) \epsilon^{h n} \\
& =\chi(n) \sum_{h=1}^{p-1} \bar{\chi}(h) \epsilon^{h}=\chi(n) \tau(\bar{\chi}) .
\end{aligned}
$$

The formula holds also for $p \mid n$, since $\chi(n)=0$ for $p \mid n$ and

$$
\sum_{h=1}^{p-1} \bar{\chi}(h)=0 \text {. }
$$

Thus

$$
\begin{aligned}
\tau(\bar{\chi}) \sum_{a=0}^{A} \sum_{n=-a}^{a} \chi(n) & =\sum_{h=1}^{p-1} \bar{\chi}(h) \sum_{a=0}^{A} \sum_{n=-a}^{a} \epsilon^{h n} \\
& =\sum_{h=1}^{p-1} \bar{\chi}(h)\left(\frac{\sin (A+1) \pi h / p}{\sin \pi h / p}\right)^{2} .
\end{aligned}
$$

${ }^{4}$ See, for example, Landau loc. cit., vol. 1, pp. 83-87. 
Consequently

$$
\begin{aligned}
p^{1 / 2}\left|\sum_{a=0}^{A} \sum_{n=-a}^{a} \chi(n)\right| & \leqq \sum_{h=1}^{p-1}\left(\frac{\sin (A+1) \pi h / p}{\sin \pi h / p}\right)^{2} \\
& =\sum_{h=1}^{p-1} \sum_{a=0}^{A} \sum_{n=-a}^{a} \epsilon^{h n} \\
& =\sum_{a=0}^{A} \sum_{n=-a}^{a}\left(\sum_{h=1}^{p} \epsilon^{h n}-1\right) \\
& =(A+1) p-(A+1)^{2} .
\end{aligned}
$$

Lemma 2. Let $p>2,1 \leqq A<(p-1) / 2$. Then, for each non-principal character, $\bmod p$, we have

$$
\frac{1}{A+1}\left|\sum_{a=0}^{A} \sum_{n=A+1-a}^{A+1+a} \chi(n)\right| \leqq p^{1 / 2}-\frac{A+1}{p^{1 / 2}} .
$$

Proof. As in Lemma 1, we have

$$
\begin{aligned}
p^{1 / 2}\left|\sum_{a=0}^{A} \sum_{n=A+1-a}^{A+1+a} \chi(n)\right| & =\left|\sum_{h=1}^{p-1} \bar{\chi}(h) e^{2 \pi i h(A+1) p}\left(\frac{\sin (A+1) \pi h / p}{\sin \pi h / p}\right)^{2}\right| \\
& \leqq \sum_{h=1}^{p-1}\left(\frac{\sin (A+1) \pi h / p}{\sin \pi h / p}\right)^{2} \\
& =(A+1) p-(A+1)^{2} .
\end{aligned}
$$

LEMMA 3. Let $p>2$. If $n$ is not a primitive root, $\bmod p$, then

$$
\sum_{k \mid p-1} \frac{\mu(k)}{\phi(k)} \sum_{\chi^{(k)}} \chi^{(k)}(n)=0,
$$

where $\chi^{(k)}$ runs over all characters $\chi$ satisfying the condition that $k$ is the least positive integer such that $(\chi)^{k}$ is the principal character.

(See Landau, loc. cit., p. 496. The condition $1 \leqq n<p$ there mentioned is not necessary.)

Theorem 1. We have $|h(p)|<2^{m} p^{1 / 2}$.

Proof. Let $p>2$. By Lemma 3, we have

$$
0=\sum_{k \mid p-1} \frac{\mu(k)}{\phi(k)} \sum_{\chi^{(k)}} \sum_{a=0}^{|h(p)|-1} \sum_{n=-a}^{a} \chi^{(k)}(n) .
$$

For $k=1$, the right-hand side gives 


$$
\begin{aligned}
\sum_{a=0}^{|h(p)|-1} \sum_{n=-a}^{a} \chi^{(1)}(n) & =\sum_{a=0}^{|h(p)|-1} 2 a . \\
& =|h(p)|^{2}-|h(p)| .
\end{aligned}
$$

On the other hand, for $k \neq 1$, we have, by Lemma 1 with $A=|h(p)|-1$,

$$
\left|\sum_{a=0}^{|h(p)|-1} \sum_{n=-a}^{a} \chi^{(k)}(n)\right| \leqq|h(p)| p^{1 / 2}-\frac{|h(p)|^{2}}{p^{1 / 2}} .
$$

Therefore

$$
\begin{aligned}
|h(p)|^{2}-|h(p)| & \leqq\left(|h(p)| p^{1 / 2}-\frac{|h(p)|^{2}}{p^{1 / 2}}\right) \sum_{k \mid p-1} \frac{|\mu(k)|}{\phi(k)} \phi(k) \\
& =2^{m}\left(|h(p)| p^{1 / 2}-\frac{|h(p)|^{2}}{p^{1 / 2}}\right) .
\end{aligned}
$$

Then

$$
|h(p)| \leqq \frac{2^{m} p^{1 / 2}+1}{1+2^{m} / p^{1 / 2}}<2^{m} p^{1 / 2} .
$$

Corollary. For $p \equiv 1(\bmod 4)$, we have $g(p)=|h(p)|<2^{m} p^{1 / 2}$.

Proof. We have to show that $|h(p)|$ is a primitive root. Suppose it is not. Then $-|h(p)|$ is a primitive root and $|h(p)|$ belongs to an exponent $l$ where $l \mid(p-1)$ and $l<p-1$, that is,

$$
\begin{aligned}
|h(p)|^{l} & \equiv 1(\bmod p), \\
(h(p))^{2 l} & \equiv 1(\bmod p) .
\end{aligned}
$$

Thus $2 l=p-1$ and $|h(p)|(p-1) / 2 \equiv 1(\bmod p)$ so that $|h(p)|$ is a quadratic residue. Since -1 is a quadratic residue, $\bmod p,-|h(p)|$ is also a quadratic residue and $\{-|h(p)|\}^{(p-1) / 2} \equiv 1(\bmod p)$. This contradicts the fact that $-|h(p)|$ is a primitive root.

REMARK. Sometimes Theorem 1 may be improved by the fact that

$$
\sum_{n=-a}^{a} \chi^{(k)}(n)=0
$$

for $\chi^{(k)}(-1)=-1$ and hence $\chi^{(k)}(n)=-\chi^{(k)}(-n)$. Thus for $p \equiv 3(\bmod 4)$,

$$
|h(p)|<2^{m-1} p^{1 / 2} .
$$

In fact, we have $g^{(p-1) / 2} \equiv-1(\bmod p)$ and $\chi^{(k)}(g)=e^{2 \pi i \lambda / k}$. Since

$$
-1=\chi^{(k)}\left(g^{(p-1) / 2}\right)=e^{\pi i(p-1) \lambda / k},
$$


we have $2 \nmid(p-1) \lambda / k$. The terms appearing in the formula of Lemma 3 are those with square-free $k$. Thus $\chi^{(k)}(-1)=-1$ holds only for the case $p \equiv 3(\bmod 4)$, and $2 \nmid \lambda$. Thus

$$
\sum_{a=0}^{|h(p)|-1} \sum_{n=-a}^{a} \chi^{(k)}(n)=0 \quad \text { for } 2 \mid k .
$$

Therefore

Then

$$
\begin{aligned}
|h(p)|^{2}-|h(p)| & \leqq\left(|h(p)| p^{1 / 2}-\frac{|h(p)|^{2}}{p^{1 / 2}}\right) \sum_{k \mid(p-1) / 2}|\mu(k)| \\
& =2^{m-1}\left(|h(p)| p^{1 / 2}-\frac{|h(p)|^{2}}{p^{1 / 2}}\right) .
\end{aligned}
$$

$$
|h(p)| \leqq \frac{2^{m-1} p^{1 / 2}+1}{1+2^{m-1} / p^{1 / 2}}<2^{m-1} p^{1 / 2} .
$$

Theorem 2. We have $g(p)<2^{m+1} p^{1 / 2}$.

Proof. Let $A$ be the greatest integer not exceeding $(g-1) / 2$. Then

$$
0=\sum_{k \mid p-1} \frac{\mu(k)}{\phi(k)} \sum_{\chi^{(k)}} \sum_{a=0}^{A} \sum_{n=A+1-a}^{A+1+a} \chi^{(k)}(n) .
$$

For $k=1$, the right-hand side gives

$$
\sum_{a=0}^{A} \sum_{n=A+1-a}^{A+1+a} \chi^{(1)}(n)=\sum_{a=0}^{A}(2 a+1)=(A+1)^{2} .
$$

For $k \neq 1$, we have

$$
\left|\sum_{a=0}^{A} \sum_{n=A+1-a}^{A+1+a} \chi^{(k)}(n)\right| \leqq(A+1) p^{1 / 2}-\frac{1}{p^{1 / 2}}(A+1)^{2} .
$$

Therefore, as in the proof of Theorem 1, we have

that is,

$$
\begin{aligned}
& (A+1)^{2}<2^{m}\left((A+1) p^{1 / 2}-\frac{1}{p^{1 / 2}}(A+1)^{2}\right), \\
& (g-1) / 2<A+1 \leqq \frac{2^{m} p^{1 / 2}}{1+2^{m} / p^{1 / 2}},
\end{aligned}
$$

$$
g \leqq \frac{2^{m-1} p^{1 / 2}}{1+2^{m} / p^{1 / 2}}+1<2^{m+1} p^{1 / 2} .
$$

National Tsing Hua University 